\title{
Isolation and characterization of lymphatic endothelial cells from human glossal lymphangioma
}

\author{
LANYING YOU*, MINGFU WU*, YING CHEN, GANG XU, JUNCHENG WEI, QIONG LI, \\ ANPING SONG, LIANGPIN ZHAO, SHUANG LI, ZHIQIANG HAN, JIANFENG ZHOU, \\ YUNPIN LU, SHIXUAN WANG, DING MA and LI MENG \\ Cancer Biology Research Center, Tongji Hospital, Tongji Medical College, Huazhong \\ University of Science and Technology, Wuhan, Hubei 430030, P.R. China
}

Received May 5, 2009; Accepted July 28, 2009

DOI: 10.3892/or_00000611

\begin{abstract}
Abnormal lymphangiogenesis is associated with several diseases such as tumor metastasis and lymphangioma. Human lymphangioma originated from the transformation of lymphatic endothelium is a benign malformation of lymphatic vessels and its pathogenesis has up to date not been illuminated and its cell model has also not been established. An optimized method was used to isolate lymphatic endothelial cells from human glossal lymphangioma (GL-LECs) and GL-LECs were further primarily cultured and expanded. GL-LECs were of typical cobblestone appearance when they reached confluence. The Weible-palade body was observed in the GL-LECs cytoplasm. Almost all GL-LECs were strongly positive for specific lymphatic markers FLT-4, LYVE-1 and prox-1 by immunocytochemistry. Furthermore, threedimension tube-like capillaries of GL-LECs resembled the lymphatic system in vivo, and the GL-LECs spheroids sprouted radically out to form three-dimensional buds when embedded in the cultured BME. These results indicated that high purity GL-LECs were successfully isolated and expanded. They had the abilities of tube formation and differentiation in vitro, which provide a favorable cell model for further uncovering the pathogenesis of human lymphangiomas.
\end{abstract}

Correspondence to: Dr Ding Ma and Dr Li Meng, Cancer Biology Research Center, Tongji Hospital, Tongji Medical College, Huazhong University of Science and Technology, Wuhan, Hubei 430030, P.R. China

E-mail: dingma424@yahoo.com; limeng@tjh.tjmu.edu.cn

${ }^{*}$ Contributed equally

Abbreviations: GL-LECs, lymphatic endothelial cells from human glossal lymphangioma; FLT-4, fms-like tyrosine kinase 4; LYVE-1, lymphatic endothelial hyaluronan receptor; TEM, transmission electron microscope

Key words: human glossal lymphangioma, lymphatic endothelial cells, LYVE-1

\section{Introduction}

The lymphatic system is made up of an extensive network of lymphatic capillaries, with functions of collecting interstitial fluid, macromolecules and even pointing leukocytes or tumor cells into the circulation system. Therefore, it plays a pivotal role in mediating tissue fluid homeostasis, tumor metastasis and immunological functions. Although the lymphatic system was discovered about 300 years ago, these functions have just been investigated in recent years due to the lack of the specific lymphatic markers. Recently, fms-like tyrosine kinase 4 (FLT-4), lymphatic endothelial hyaluronan receptor (LYVE-1), Prox-1, and podoplanin have been identified as the specific lymphatic endothelial cell markers and largely restricted to lymphatics in adult tissues (1-6), which provided new insights into the molecular mechanisms of lymphatics in physiologic functions and pathologic processes such as inflammations, tumor metastasis, lymphedema and lymphangioma. Human lymphangioma, which is a kind of benign tumor originated from lymphatic vessels, is largely diagnosed in children and has always been defined as congenital malformations. Although human lymphangiomas are asymptomatic in the early course, they develop and have characteristics of progressive growth, compression and infiltration into adjacent tissues. The most acceptable therapy for human lymphangioma is surgery at present, but it has many limitations such as the risk of complications and recurrence. The limitations of surgical treatment have awoken the interests in other therapeutic methods. The administration of sclerosing agents such as bleomycin and hypotonic salt solutions provokes an inflammation of the vascular endothelium leading to total or partial remission of human lymphangioma (7-11). These substances may extend beyond the limits of the original lymphangioma, unsatisfactory aesthetic results and increased difficulties for future surgery. As a result, a more innovative and effective treatment is highly expected. However, the pathogenesis of human lymphangioma has not been elucidated for further investigation primarily due to lack of pathological research models. Accordingly, it is imminent and necessary to construct a cell model of human lymphangioma for in vitro study of its molecular events via isolating and expanding lymphatic endothelial cells from human glossal lymphangiomas (GL-LECs). 
Early in 1980s, investigators tried to isolate lymphatic endothelial cells from several kinds of animal macrolymphatics including rat and bovine thoracic lymph duct, mesentery lymphatics by collagenase digestion method. Formerly, it was difficult to sort lymphatic endothelial cells from tissues because of many different types of cells such as mesenchymocytes and vascular endothelial cells. Increasing findings of specific lymphatic endothelial cell markers make it feasible to isolate pure LECs from human normal tissues for further study. Podgrabinska et al (11) and Mäkinen et al (1) used LYVE-1 antibody or VEGFR-3 antibody coated magnetic beads to sort LYVE-1 or VEGFR-3 positive lymphatic endothelial cells from dermal cell mixtures, respectively. Kriehuber et al (12) used multicolor flow cytometry to sort podoplanin positive lymphatic endothelial cells from human dermal cell suspensions. However, there were no pathological cell models to further study the pathogenesis of lymphatic associated diseases. Therefore, in the present study an optimized collagenase digestion method was used to isolate pure human glossal lymphangiomaassociated LECs based of the fact that there are plenty of dilated lymphatics in human lymphangioma specimens, and then the primarily cultured cell lines were obtained. Molecular phenotypes and functions of glossal lymphangioma-associated LECs were further characterized by immunohistochemistry staining, tube-formation assay and sprouting assay.

\section{Materials and methods}

Mainly materials. M199 culture medium, Dulbecco's modified Eagle's medium (DMEM) and fetal bovine serum were purchased from Gibco Co. (USA). Collagenase and collagen type I were obtained from Sigma Co. (Germany). Mouse anti human FLT-4 monoclonal antibody was from Santa Cruz (USA), rabbit anti-human LYVE-1 polyclonal antibody was from R\&D Co. (USA), and rabbit antihuman Prox-1 polyclonal antibody from Abcam Co. (UK). Cultrex Basement Membrane Extract (BME) was obtained from Invitrogen Inc. (USA). Vascular endothelial growth factor-C (VEGF-C) was purchased from BD Co. (USA). The immunostaining envision systems were purchased from Dako (USA).

Specimen preparing. Human glossal lymphangioma specimens were obtained from Wuhan Oral Medical Hospital affiliated to Wuhan University. After surgical removal, in less than $45 \mathrm{~min}$, the fresh tissues were divided into two pieces, the small one was fixed in $4 \%$ paraformaldehyde for preparing paraffin blocks, the larger one was immediately put in cold sterile M199 medium supplemented with fungizone (10 $\mu \mathrm{g} / \mathrm{ml})$, penicillin $(100 \mu / \mathrm{ml})$, streptomycin $(100 \mu \mathrm{g} / \mathrm{ml})$ and $10 \%$ fetal bovine serum and then transferred it to our laboratory for further investigation.

Immunohistochemistry. Immunohistochemistry was used to identify the pathological characteristics of human lymphangioma (13), paraffin-embedded sections were produced from human glossal lymphangioma specimens fixed in $4 \%$ paraformaldehyde. Sections were deparaffinized in $100 \%$ xylene twice, followed by gradient ethanol $(100,95,90,80$ and $70 \%$ ). To expose the antigen epitopes completely, the sections were treated in a microwave in the presence of citrate buffer $(\mathrm{pH}$ 6.0) three times, and then incubated with primary antibody overnight at $4^{\circ} \mathrm{C}$ all diluted 1:200). Remaining steps were conducted as the procedure of the staining kit.

Isolation and primary culture of GL-LECs. The tissues were washed with Hank's solution supplemented with the antimicrobial agents as above, and the connective tissues were removed, and then the tissue block was finely minced with scissors into $0.5 \times 0.5 \times 0.5 \mathrm{~mm}$ size pieces and subjected to enzymatic digestion for $1 \mathrm{~h}$ at $37^{\circ} \mathrm{C}$ with $0.25 \%(\mathrm{w} / \mathrm{v})$ collagenase/serum free DMEM. The digestion product was filtered through a $30 \mathrm{~mm}$ pore size filter and the filtering product was centrifuged at 1,200 rpm for $10 \mathrm{~min}$, washed the cells with Hank's solution twice and centrifuged, then resuspended the cells with complete medium EGM (endothelial growth medium, M199 medium, supplemented with $20 \%$ fetal bovine serum, VEGF-C $100 \mu \mathrm{g} / \mathrm{ml}$, heparin $40 \mathrm{U} / \mathrm{ml}$, penicillin $100 \mathrm{U} / \mathrm{ml}$ and streptomycin $100 \mu \mathrm{g} / \mathrm{ml}$ ) and transferred them to T25 flask and cultured in incubator. Next day, non-adherent cells were removed and discarded, whereas the adherent ones were washed three times with Hank's solution with antibiotics, and incubated with EGM until confluence. At about $80-90 \%$ confluence, the cells were digested for subculture with $0.25 \%$ trypsinase for 3-5 min at $37^{\circ} \mathrm{C}$. The digested cells were transferred to a new T25 culture flask. Cell culture media were replaced 2-3 times per week. The isolated LECs can be cultured at least 10 passages and can be frozen in liquid nitrogen with $20 \%$ fetal bovine serum and $10 \%$ DMSO.

\section{Identification of GL-LECs}

Morphology and ultrastructure of GL-LECs. The morphology and ultrastructure of GL-LECs were observed under a light microscope (Olympus, Japan) and transmission electron microscope (TEM) (FEITecnaiG ${ }^{2}$ type 12, Holland), respectively. Cells were harvested with trypsinase digestion, centrifuged and washed in phosphate buffer solution $(0.1 \mathrm{M}$, $\mathrm{pH} 7.4$ ), and then fixed with $2.5 \%$ glutaraldehyde and postfixed in osmium tetroxide. The samples were dehydrated with gradient ethanol 50, 70, 95 and 100\% ethanol and propylene oxide prior to embedding in epoxy. The grids were stained with lead citrate and uranyl acetate. The samples were observed under transmission electron microscope (TEM), and photographed.

Immunocytochemistry of GL-LECs. Immunocytochemistry method was used to further identify the GL-LECs by detecting the expression of several special lymphatic endothelial cell markers. GL-LECs were seeded on cover slips overnight and fixed in $4 \%$ paraformaldehyde at room temperature. After washing twice in PBS, the cells coated on the cover slips were incubated with primary antibody overnight at $4^{\circ} \mathrm{C}$ all diluted 1:100). Negative control, in which cells were treated with PBS instead of primary antibody, was also used. 


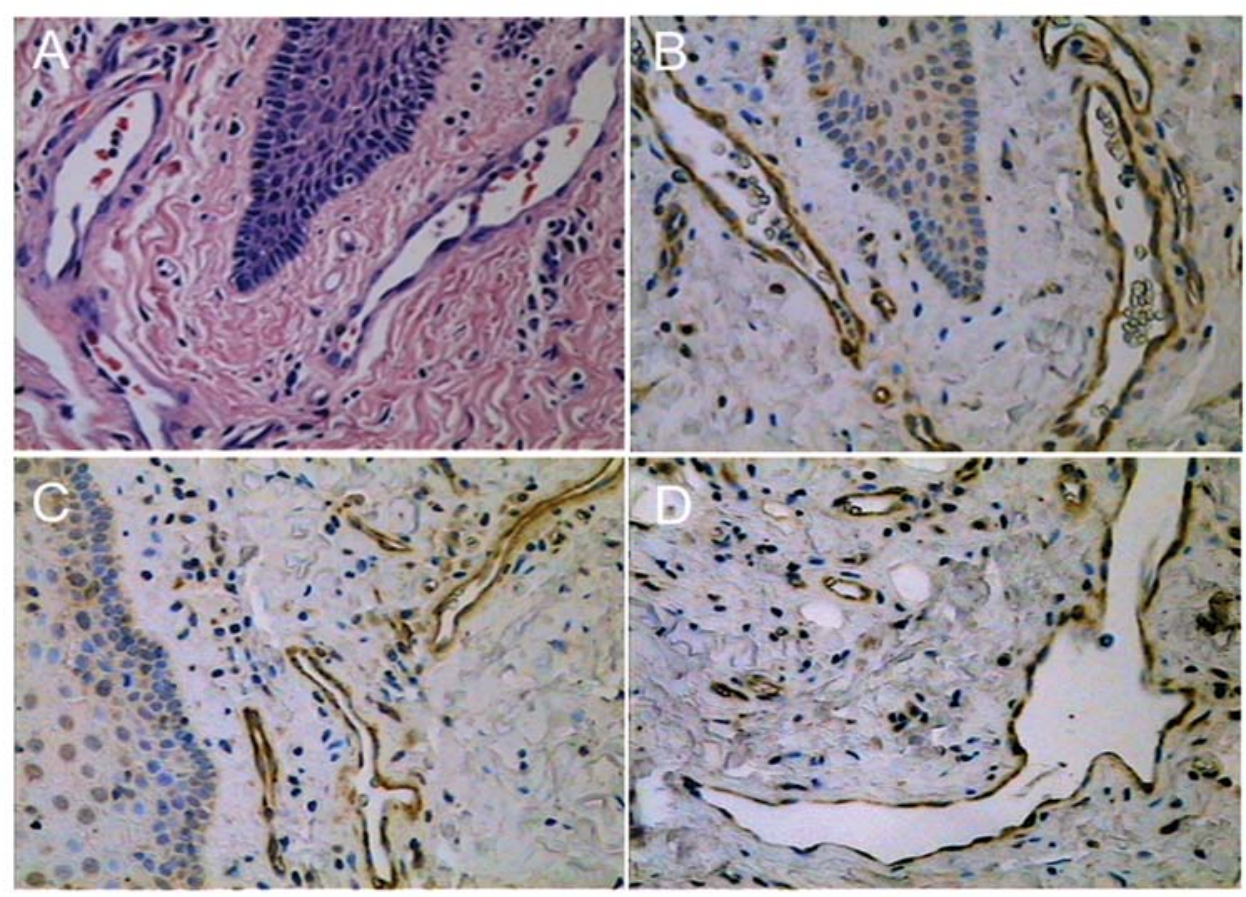

Figure 1. Characteristics of human glossal lymphangioma by immnunohistochemistry. (A) H\&E staining showed that there are considerable number of dilated vessels in the tissue sections of human glossal lymphangioma. (B-D) dilated vessels in the tissue sections were positive for FLT-4, LYVE-1 and prox-1 on endothelial cell membrane and cell nucleus, respectively. Original magnification, x100.

Tube formation assay and spouting assay in vitro. Tube formation assay was performed as described (14). Cells were harvested with trypsinase. A collagen stock solution was prepared prior to use by mixing collagen type I (Sigma, equilibrated to $2 \mathrm{mg} / \mathrm{ml}, 4^{\circ} \mathrm{C} ; 8$ vol.) with $10 x$ DMEM (1 vol.) and $0.1 \mathrm{~N} \mathrm{NaOH}$ (approx. 1 vol.) to adjust the $\mathrm{pH}$ to 7.4. The solution was allowed to gel for $30 \mathrm{~min}$ at $37^{\circ} \mathrm{C}$. Then LECs were seeded on the gel and cultured in incubator at $37^{\circ} \mathrm{C}$. Cells cultured in the gel were observed for $48 \mathrm{~h}$ under a contrast phase microscope. After at least one week, Collagen gels were fixed in $2.5 \%$ glutaraldehyde and cut into pieces, washed in phosphate buffer $(0.1 \mathrm{M}, \mathrm{pH} 7.4)$ and postfixed in osmium tetroxide, dehydrated in a graded series of ethanol, and embedded in epon. Ultrathin $(50-80 \mathrm{~nm})$ sections were cut and collected on copper grids, stained with uranyl acetate and lead citrate for observation by TEM. Sprouting assay was performed according to protocols in the literature $(15,16)$. Cells (800 per well) were seeded in $0.24 \%$ methylcellulose M199 medium for overnight incubation to form a single spheroid. Spheroids were collected, centrifuged at low speed, and seeded in BME (Trevigen Inc., USA). BME was prepared prior to use by diluted with DMEM containing $0.5 \%(\mathrm{w} / \mathrm{v})$ carboxymethylcellulose at $1: 1$. The spheroid-containing gel was rapidly transferred into pre-warmed 24-well plates and allowed to polymerize (for $1 \mathrm{~min}$ ), after which $0.15 \mathrm{ml}$ EGM basal medium was pipetted on the top of the gel. Sprout outgrowth was observed for $24 \mathrm{~h}$ under a contrast phase microscope.

\section{Results}

Characteristics of human glossal lymphangioma. In the present study, H\&E staining and immunohistochemistry analysis were used to identify the pathological feature of human glossal lymphangioma. H\&E staining revealed the fact that there were plenty of dilated vessels in the human glossal lymphangioma sections (Fig. 1A). Specific lymphatic cell markers FLT-4, LYVE-1 and Prox-1 were used to show that dilated vessels in the sections were lymphatics. The results indicated that the dilated vessels in the serial sections of the same tissues, which were positive for FLT-4 and LYVE-1 in endothelial cell membrane or cytoplasm and Prox-1 in cell nucleus, were identified as lymphatic (Fig. 1B-D) and agreed with previous reports that human lymphangiomas are rich in dilated lymphatics (17). It is evident that human glossal lymphangioma provides an available and reliable source of LECs for further isolation.

Isolation and primary culture of GL-LECs. The fresh tissues from human glossal lymphangiomas were processed and subjected to collagenase digestion. The periphery of the neoplasm was sheared absolutely to keep the target cells from mixing with glossal tissue cells. Bacteria and fungi adhering to their surface were removed by several washings in a solution containing high concentrations of penicillin, streptomycin and fungizone. The non-adherent cells were removed with washing solution next day and the adherent ones obtained were the primary culture cells. The population of primary culture cells was few, and the cells grew slowly as an adherent monolayer. As observed under light microscope, cells were fusiform or polygon shaped and were uniform in size, and the cell nuclei were clear and orbicular-ovate (Fig. $2 \mathrm{~A})$. The cell populations were propagated persistently for about two weeks until complete confluence. Cells were propagated with $0.025 \%$ trypsin at $80-90 \%$ confluence. The sub-cultured cells grew much quicker and reached 

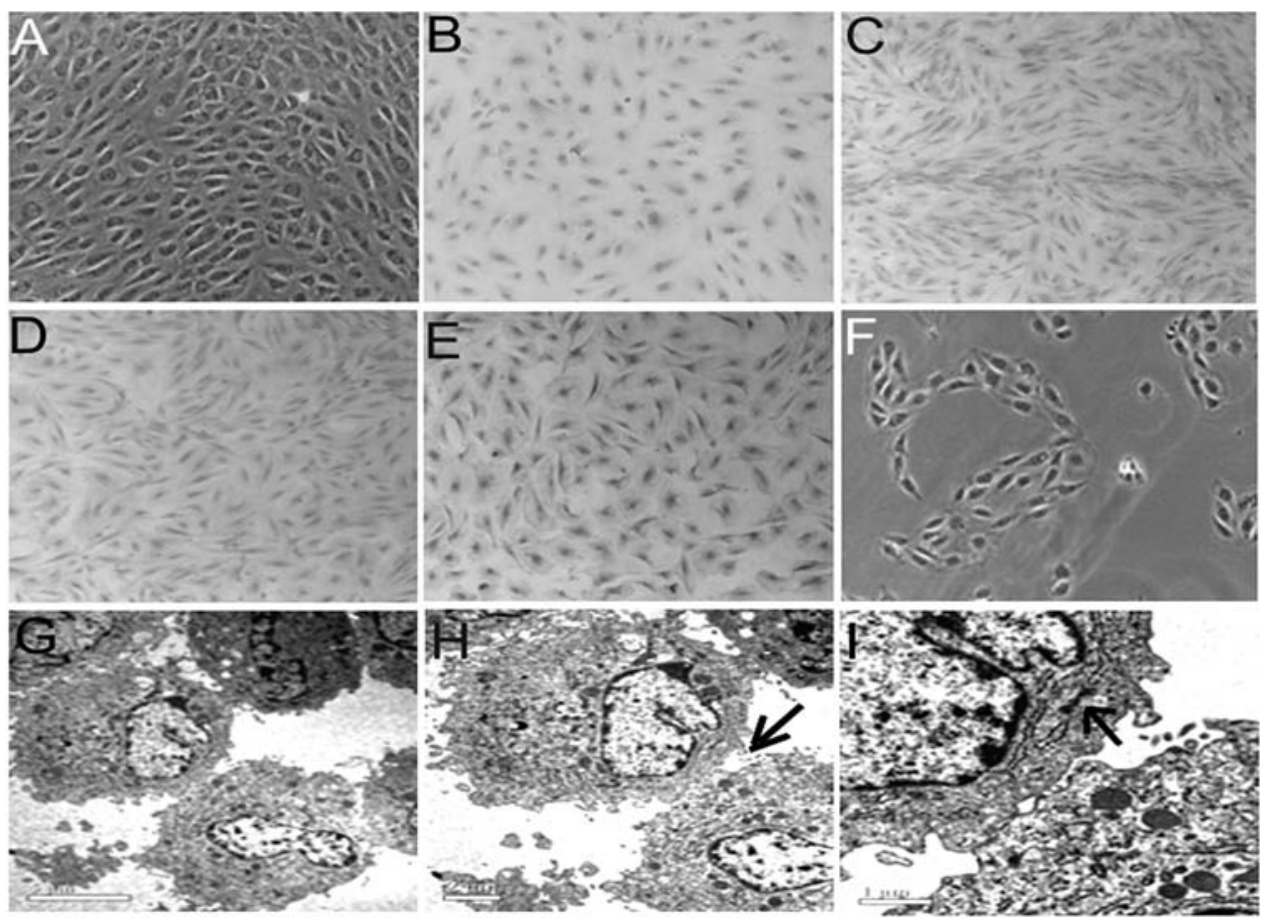

Figure 2. Identification of isolated and primarily cultured GL-LECs. (A) Phase contrast photomicrograph showed the growth pattern of conglobation and a typical cobblestone appearance when cells reached confluence. (B) Negative control and cells nucleus were counterstained with hematoxylin. (C-E) Immnuocytochemistry analysis indicated that in isolated and primarily cultured GL-LECs, almost all GL-LECs were positive for FLT-4 and LYVE-1 on cell membrane and cytoplasm and prox-1 were shown strongly positive in GL-LECs nucleus. (F) In GL-LECs, the central cells were micro size and close-up, however, the adjacent ones were macro size and irregular, and several spindle-shaped cells were seen in the periphery (original magnification, $\mathrm{x} 20$ ). (G-I) Transmission electron micrograph image of GL-LECs. (G) Human LECs emerged in scale-shape and appeared as continuous monocellular layer. (H) Intercellular gap junction appeared in adjacent LECs, indicated by an arrow. (I) a higher magnification of the same region in the panel $\mathrm{H}$ is shown by the arrow. Under high power lens, the organoids such as Weible-palade body (shown by the arrow I), endocytoplasmic reticulum, plasmalemmal vesicle and cytoplasmic processes were observed transparently. [bar in $(\mathrm{G})=5 \mu \mathrm{m}$; bar in $(\mathrm{H})=2 \mu \mathrm{m}$ and bar in $(\mathrm{I})=1 \mu \mathrm{m})]$.

confluence in about 7 days, they showed typical cobblestone morphology, a specific characteristic of endothelial cells (Fig. 2A). When propagated up to confluence, the growth rate of GL-LECs slowed down, and the typical contact inhibition phenomenon of endothelial cell growth emerged.

Identification of GL-LECs. Ultrastructure of GL-LECs was observed by transmission electron microscopy. GL-LECs emerged scale-shaped and with monocellular layer appearance under TEM (Fig. 2G). The majority of cells existed in conglobation, so they were discriminated from the fibroblasts which always exist in sporadic form. Gap or tight inter-cellular junctions between consecutive GL-LECs were frequently overviewed (Fig. 2H). Weibel-palade body, a typical granulation of endothelial cells was observed in cytoplasm in rod-shape in the longitudinal section with high electron density (Fig. 2I). Other specific endothelial cell organoids such as endocytoplasmic reticulum, plasmalemmal vesicle and cytoplasmic processes were also viewed in the cytoplasm. Although the TEM images demonstrated typical endothelial cell characteristics, lymphatic endothelial cells and blood vascular endothelial cells share common features in these ultra structures. Therefore, to further identify the GL-LECs, the immunocytochemistry method was used to detect the expression of several special lymphatic endothelial cell markers in GL-LECs. Almost all the cells were positive for FLT-4 and LYVE-1 in cell membrane and cytoplasm, and strongly positive for Prox-1 in the cell nucleus (Fig. 2C-E), indicating that the cells isolated from human glossal lymphangioma were the lymphatic endothelial cells with more than $95 \%$ purity.

Functional features in vitro of GL-LECs. In order to reveal the function properties of cultured GL-LECs in vitro, tube formation assay and sprouting assay were used to observe the ability of lymphatic formation and cell differentiation, respectively. When subjected to tube-forming assay on collagen type I gel, GL-LECs began to form tube-like structures in the conditions of EGM added with VEGF-C after $48 \mathrm{~h}$ of incubation. When cultured in the gel longer, GL-LECs advanced to form capillary-like tubes in very different directions, and the tubes from different directions linked up to form a complex lymphatic vascular pluex, which is similar with the lymphatic system in vivo (shown in Fig. $3 \mathrm{~A}$ and $\mathrm{B}$ ). The tube-like construction ultrastructure was further verified by TEM. Cross sections of collagen gels revealed true capillary lumens and their walls were composed of lymphatic endothelial cells lacking continuous basal lamina, and then lymphatic endothelial cells typically overlapping intercellular junction was also seen in the section (shown in Fig. 3D and E). The GL-LEC spheroids formed after the cells were seeded in $0.24 \%$ methylcellulose for 

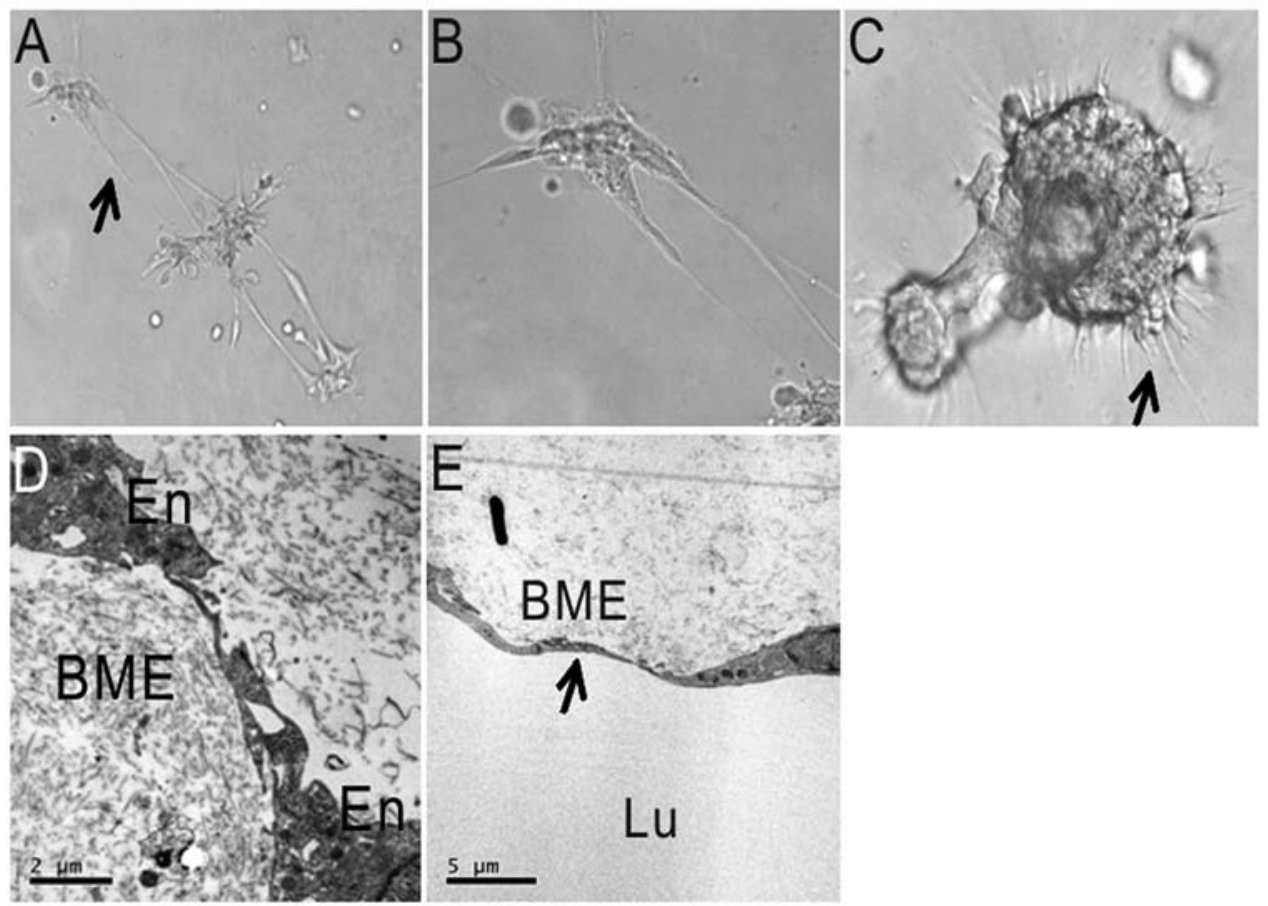

Figure 3. The abilities of differentiation and tube formation in vitro of human GL-LECs. (A) Human GL-LECs formed 3-dimensional capillary-like tubes and the tubes linked to each other to form a closed network. (B) A higher magnification of a certain region in the picture A. (C) in the cutlery of BME, human GLLECs sprouted radically out of spheroplasts and formed 3-dimensional buds. (D and E) the ultrastructure of capillary-like tubes formed by human GL-LECs in collagen gel I was observed by TEM. (D) Showed a true lumen composed by several human GL-LECs. (E) Revealed a typical overlapping intercellular junction (indicated by an arrow). Lu, lumen; BME, basement membrane extracts; En, human endothelial cells.

about $24 \mathrm{~h}$. The gel-embedded GL-LECs spheroids sprouted radically for the first $48 \mathrm{~h}$ and formed 3-dimensional buds in BME (shown in Fig. 3C). These results indicated that the abilities of differentiation and tube formation in vitro were conferred to GL-LECs.

\section{Discussion}

In the present study, lymphangioma-associated LECs isolated by an optimized method could be primarily cultured and expanded more than 10 passages. Furthermore, lymphangioma-associated LECs were identified to characterize the common features of the reported LECs. Thus, we successfully developed a cell model of lymphatic endothelium for further investigating the molecular mechanism of the pathogenesis of human lymphangiomas.

Hyperplasia or abnormal growth of lymphatic vessels is associated with lymphangiomas. Lymphangiomas have been reported with the characteristics of numerous dilated lymphatic vasculatures or over-proliferation of lymphatic vessels $(18,19)$. Although increasing cases of lymphangiomas were reported, the molecular events such as the genetic and proteomic changes are not defined. Only recently, the coexpression of VEGF and VEGFR in 29 cases of human lymphangioma were detected by RNA in situ hybridization, suggesting that VEGF-C and its receptors may actively participate in the formation of human lymphangioma by autocrine or paracrine regulation $(19,20)$. However, although mouse lymphatic endothelial cells were isolated and characterized $(20,21)$, there is no previous cell model of human LECs to discover the molecular regulation of lymphangiogenesis in lymphatic-associated disorders. In the present study, human glossal lymphangioma was selected to be the disease model for further investigation. The fresh tissue sections stained by hematoxylin-eosin showed plenty of dilated vessels without any blood cells instead of the lymph fluid. To further confirm the fact that the dilated vessels were lymphatics, the expressions of special lymphatic endothelial cell markers such as FLT-4, LYVE-1 and prox-1 were detected by immunohistochemistry staining. We found that almost all of the dilated vessels were positive for FLT-4, LYVE-1 and prox-1. Accordingly, it naturally assured that human glossal lymphangioma was a reliable and valuable source of human LECs.

Recently, an immunomagnetic based purification method was used to isolate LECs from mixed cultures. Magnetic beads coated by LYVE-1, podoplanin, and VEGFR-3 antibodies were applied to separate the related subpopulations from gross cells, respectively $(1,11,12)$. These methods only purified the appointed subgroups from several mixed cultures, thus this caused not only the omission of partial LECs originally included, but also partial contamination of heterogeneous cells such as blood endothelial cells. Before several novel specific lymphatic markers were reported, LECs were mainly isolated from different tissues by means of collagenase treatment. But during the primary cultures, collagenase disposal may also result in contamination of other cells including blood endothelial cells and interstitial cells. Here, we optimized the protocol of collagenase disposal. Whether we could obtain high purity of GL-LECs or not, the collage- 
nase digestion time was pivotal and a key factor. Longer digested, more contaminated cells would be contained in the primary cultures. To achieve higher purity of LECs, we utilized the fact that endothelial cells were always easier to trypsinize and existed in the pattern of conglobation (containing several endothelial cells after trypsinization) to eliminate the contaminated mesenchymal cells with serial subcultivation. Meanwhile, VEGF-C, which was recently revealed with the ability of selectively inducing survival of LECs but not blood endothelial cells, was added in the special growth medium to promote the growth and expansion of GL-LECs (11), and heparin, which enhances the growth of LECs and reduce concentrations of VEGF-C for long-term serial culture as reported by Engelmann et al (21), was also added in the culture medium to ensure permanent cell phenotype. When these measures were carried out, pure GL-LECs were expected.

Our results indicated that GL-LECs exhibited a cobblestone appearance at confluence, which was well received as a representative morphological feature of endothelial cells (22-24). Weible-palade body, a kind of specific granule of endothelial cells, was captured under TEM as reported (22). An abundance of cytoplasmic vesicles, which are one of the typical features of LECs $(4,25-27)$, were seen in the ultrathin sections from human glossal lymphangioma. In addition, gap and tight intercellular junctions in adjacent cells were also displayed by TEM. These findings obviously demonstrated that GL-LECs had the characteristics of endothelial cells in morphology and ultrastruture. Almost all GL-LECs were positive for FLT-4, LYVE-1 and prox-1. The phenotypic patterns of GL-LECs were extremely similar to those observed in human glossal lymphangioma, suggesting that GL-LECs in vitro maintained the original molecular characterizations and a considerable high purity and then in vitro kept the primal information at a maximum degree. Furthermore, the functions of GL-LECs, such as forming complex tube-like capillary networks in collagen type I gel and GL-LECs spheroids sprouting to form three-dimentional buds, simulating the lymphatic system in vivo, thus proving their capabilities to form lymphatic vessels and in vitro differentiate. Taken together, GL-LECs successfully isolated through collagenase treatment were easily cultured in EGM supplemented with VEGF-C without lack of any molecular phenotype and functional activities in vitro.

In summary, human glossal lymphangioma provides an available source of human LECs. A convenient, facile and reliable method has successfully been developed to isolate and expand human LECs in vitro. GL-LECs obtained will provide a good cell model for lymphatic malformations and be useful for investigating the molecular mechanisms of human lymphangiomas.

\section{Acknowledgements}

Grant support: National Science Foundation of China (30371657; 30672227; 30770913 and 30170976) and the '973' Program of China (no. 2009CB521800).

\section{References}

1. Makinen T, Veikkola T, Mustjoki S, Karpanen T, Catimel B, Nice EC, Wise L, Mercer A, Kowalski H, Kerjaschki D, Stacker SA, Achen MG and Alitalo K: Isolated lymphatic endothelial cells transduce growth, survival and migratory signals via the VEGF-C/D receptor VEGFR-3. EMBO J 20: 4762-4773, 2001.

2. Nightingale TD, Frayne ME, Clasper S, Banerji S and Jackson DG: A mechanism of sialylation functionally silences the hyaluronan receptor LYVE-1 in lymphatic endothelium. J Biol Chem (In press).

3. Prevo R, Banerji S, Ferguson DJ, Clasper S and Jackson DG: Mouse LYVE-1 is an endocytic receptor for hyaluronan in lymphatic endothelium. J Biol Chem 276: 19420-19430, 2001 .

4. Johnson NC, Dillard ME, Baluk P, McDonald DM, Harvey NL, Frase SL and Oliver G: Lymphatic endothelial cell identity is reversible and its maintenance requires Prox 1 activity. Genes Dev 22: 3282-3291, 2008.

5. Costa da Cunha Castro E and Galambos C: Prox-1 and VEGFR3 antibodies are superior to D2-40 in identifying endothelial cells of lymphatic malformations-a proposal of a new immunohistochemical panel to differentiate lymphatic from other vascular malformation. Pediatr Dev Pathol (In press).

6. Wetterwald A, Hoffstetter W, Cecchini MG, Lanske B, Wagner C, Fleisch $\mathrm{H}$ and Atkinson $\mathrm{M}$ : Characterization and cloning of the E11 antigen, a marker expressed by rat osteoblasts and osteocytes. Bone 18: 125-132, 1996.

7. Lee BB, Kim YW, Seo JM, Hwang JH, Do YS, Kim DI, Byun HS, Lee SK, Huh SH and Hyun WS: Current concepts in lymphatic malformation. Vasc Endovascular Surg 39: 67-81, 2005.

8. Molitch HI, Unger EC, Witte CL and van Sonnenberg E: Percutaneous sclerotherapy of lymphangiomas. Radiology 194: 343-347, 1995

9. Souza RJ and Tone LG: Treatment of lymphangioma with alpha-2a-interferon. J Pediatr (Rio J) 77: 139-142, 2001.

10. Sanlialp I, Karnak I, Tanyel FC, Senocak ME and Buyukpamukcu N: Sclerotherapy for lymphangioma in children. Int J Pediatr Otorhinolaryngol 67: 795-800, 2003.

11. Podgrabinska S, Braun P, Velasco P, Kloos B, Pepper MS and Skobe M: Molecular characterization of lymphatic endothelial cells. Proc Natl Acad Sci USA 99: 16069-16074, 2002.

12. Kriehuber E, Breiteneder-Geleff S, Groeger M, Soleiman A, Schoppmann SF, Stingl G, Kerjaschki D and Maurer D: Isolation and characterization of dermal lymphatic and blood endothelial cells reveal stable and functionally specialized cell lineages. J Exp Med 194: 797-808, 2001.

13. Li Q, Wu M, Wang H, Xu G, Zhu T, Zhang Y, Liu P, Song A, Gang C, Han Z, Zhou J, Meng L, Lu Y, Wang S and Ma D: Ezrin silencing by small hairpin RNA reverses metastatic behaviors of human breast cancer cells. Cancer Lett 261: 55-63, 2008.

14. Maruyama K, Ii M, Cursiefen C, et al: Inflammation-induced lymphangiogenesis in the cornea arises from $\mathrm{CD} 11 \mathrm{~b}$ positive macrophages. J Clin Invest 115: 2363-2372, 2005.

15. Kazanskaya O, Ohkawara B, Heroult M, Wu W, Maltry N, Augustin HG and Niehrs C: The Wnt signaling regulator Rspondin 3 promotes angioblast and vascular development. Development 135: 3655-3664, 2008.

16. Korff T and Augustin HG: Tensional forces in fibrillar extracellular matrices control directional capillary sprouting. $\mathbf{J}$ Cell Sci 112: 3249-3258, 1999

17. Faul JL, Berry GJ, Colby TV, Ruoss SJ, Walter MB, Rosen GD and Raffin TA: Thoracic lymphangiomas, lymphangiectasis, lymphangiomatosis, and lymphatic dysplasia syndrome. Am J Respir Crit Care Med 161: 1037-1046, 2000.

18. Yaita T, Onodera K, Xu H and Ooya K: Histomorphometrical study in cavernous lymphangioma of the tongue. Oral Dis 13: 99-104, 2007.

19. Huang HY, Ho CC, Huang PH and Hsu SM: Co-expression of VEGF-C and its receptors, VEGFR-2 and VEGFR-3, in endothelial cells of lymphangioma. Implication in autocrine or paracrine regulation of lymphangioma. Lab Invest 81: 1729-1734, 2001.

20. Sironi M, Conti A, Bernasconi S, Fra AM, Pasqualini F, Nebuloni M, Lauri E, De Bortoli M, Mantovani A, Dejana E and Vecchi A: Generation and characterization of a mouse lymphatic endothelial cell line. Cell Tissue Res 325: 91-100, 2006. 
21. Engelmann K, Bednarz J and Bohnke M: Endothelial cell transplantation and growth behavior of the human corneal endothelium. Ophthalmologe 96: 555-562, 1999.

22. Djoneidi $\mathrm{M}$ and Brodt $\mathrm{P}$ : Isolation and characterization of rat lymphatic endothelial cells. Microcirc Endothelium Lymphatics 7: 161-182, 1991.

23. Jones BE and Yong LC: Culture and characterization of bovine mesenteric lymphatic endothelium. In Vitro Cell Dev Biol 23: 698-706, 1987.

24. Johnston MG and Walker MA: Lymphatic endothelial and smooth-muscle cells in tissue culture. In Vitro 20: 566-572, 1984.
25. Leak LV: The transport of exogenous peroxidase across the blood-tissue-lymph interface. J Ultrastruct Res 39: 24-42, 1972.

26. Leak LV: The structure of lymphatic capillaries in lymph formation. Fed Proc 35: 1863-1871, 1976.

27. Leak LV and Jones M: Lymphatic endothelium isolation, characterization and long-term culture. Anat Rec 236: 641-652, 1993. 